\title{
openheart Heart sounds: a pilot randomised trial to determine the feasibility and acceptability of audio recordings to improve discharge communication for cardiology inpatients protocol
}

\author{
Stacey L Schott, ${ }^{1}$ Michelle D Dannenberg, ${ }^{2}$ Shayne E Dodge, ${ }^{1}$ \\ Jesse A Schoonmaker, ${ }^{2}$ Molly M Caisse, ${ }^{2}$ Paul J Barr, ${ }^{2}$ A. James O'Malley, ${ }^{1}$ \\ Martha L. Bruce ${ }^{2}$
}

\begin{abstract}
- Additional material is published online only. To view, please visit the journal online (http://dx.doi.org/10.1136/ openhrt-2019-001062).
\end{abstract}

To cite: Schott SL, Dannenberg MD, Dodge SE, et al. Heart sounds: a pilot randomised trial to determine the feasibility and acceptability of audio recordings to improve discharge communication for cardiology inpatients protocol. Open Heart 2019;6:e001062. doi:10.1136/

openhrt-2019-001062

Received 1 April 2019

Revised 24 May 2019

Accepted 13 June 2019

Check for updates

C) Author(s) (or their employer(s)) 2019. Re-use permitted under CC BY-NC. No commercial re-use. See rights and permissions. Published by BMJ.

${ }^{1}$ Department of Cardiovascular Medicine, Dartmouth-Hitchcock Medical Center, Lebanon, New Hampshire, USA

${ }^{2}$ The Dartmouth Institute for Health Policy and Clinical Practice, Dartmouth College Geisel School of Medicine, Hanover, New Hampshire, USA

Correspondence to Dr Stacey L Schott; Stacey.L. Schott@hitchcock.org

Dr Paul J Barr; Paul.J.Barr@ Dartmouth.edu

\section{ABSTRACT}

Introduction Ineffective hospital discharge communication can significantly impact patient understanding, safety and treatment adherence. This may be especially true for cardiology inpatients who leave the hospital with complex discharge plans delivered in a time-pressured discharge discussion. The goal of this pilot trial was to determine if providing supplemental audiorecorded discharge instructions is feasible and to explore its impact on cardiology patients' ability to understand and self-manage their care

Methods and analysis We will conduct a parallel-group, randomised controlled trial in adult cardiology inpatients with balanced blocking by a physician. Patients $(n=50)$ will be randomised to usual care (verbal discussion and written summary) or intervention (usual care, plus audiorecorded discharge discussion provided to patients on a portable electronic recording device). Enrolled patients will complete study assessments immediately prior to the discharge discussion, immediately postdischarge discussion and 1 week after hospital discharge by telephone. Primary outcomes include the proportion of eligible providers and inpatients who agree to take part in the trial, the proportion of inpatients who receive the audio recording in accordance with a fidelity checklist, and the proportion who use the audio recording. We will analyse preliminary data about the impact of audio recording on patient activation, health confidence, provider communication ability, adherence and 30-day readmissions.

Ethics and dissemination This trial was approved by The Committee for the Protection of Human Subjects (CPHS) at Dartmouth College (CPHS\# 00031211). Findings will be disseminated in scientific journals and at meetings.

Trial registration number NCT03735342

Protocol version 1.0

\section{INTRODUCTION}

\section{Background and rationale}

Ineffective discharge communication can impact patient safety, treatment adherence

\section{Key questions}

What is already known about this subject?

- Patients are beginning to audio record clinical interactions with their healthcare providers, and systematic reviews report improvement in patients' recall, understanding and satisfaction, primarily in the outpatient setting, but the full utility of audio recordings as a tool to promote the review of discharge instructions has not been determined.

What does this study add?

- The objective of the Heart Sounds pilot study is to determine if it is feasible to apply audio recordings as an adjunctive communication technology at discharge in a rapid-turnover inpatient cardiology unit, where the risk of communication errors and misunderstandings may be high.

How might this impact clinical practice?

- If audio recordings are feasible and acceptable for complex cardiovascular patients and their providers, this intervention could be routinely offered and may have the potential to impact key patient outcomes such as understanding, self-management, adherence and readmission rates.

and readmission rates. ${ }^{12}$ Patients with cardiovascular disease, including those with congestive heart failure (CHF), dysrhythmia and acute coronary syndrome (ACS), represent a significant proportion of hospitalised patients and, as a result, are at elevated risk for discharge communication failure. ${ }^{3}$ Their discharge plans are complicated by long and dynamic medication lists, postprocedural care instructions and drastic lifestyle changes. Understanding these plans may be difficult since patients receive a large amount of technical information in a time-pressured discharge process. Studies confirm patients' 
difficulty retaining this information, especially when it is provided verbally, citing failure to remember more than $80 \%$ of what was communicated within 1 minute. When patients do recall important information, $50 \%$ is recalled incorrectly. ${ }^{4}$ Multiple complex factors, including age, health literacy, education, preferred learning style and provider communication ability, affect the retention of medical information. ${ }^{5}$ Patients who understand their discharge instructions are more likely to adhere to treatment plans and are $30 \%$ less likely to be readmitted. ${ }^{6}$ Our hospital's Hospital Consumer Assessment of Healthcare Providers and Systems (HCAPS) data corroborate the difficulty cardiology inpatients have recalling and retaining important information, indicating they routinely struggle to understand their discharge instructions compared with patients discharged from other inpatient units. This may result in difficulty managing their care at home and contributes to frequent postdischarge telephone calls ${ }^{7}$ (SL Schott, unpublished data, 2018).

Many discharge improvement plans have been attempted, ${ }^{6}$ and patients appear willing to engage in innovative information delivery strategies to help mitigate communication issues. ${ }^{8}$ One novel strategy to improve communication of discharge instructions is emerging in the form of sharing audio recordings. Reviews indicate that sharing audio-recorded clinic visits with patients, mainly in oncology and paediatrics, has resulted in improved patient recall, satisfaction and understanding. ${ }^{10}{ }^{11}$ However, the true utility of providerpatient audio recordings has yet to be determined, and its routine practice is in its infancy. To date, the feasibility of implementing this strategy to improve the delivery of critical discharge information for cardiology inpatients has not been examined. This study may serve to further understand the capability of audio recording as a quality improvement tool at the fragile time of inpatient discharge. Our long-term goal is to improve health outcomes for patients by enhancing access to their health information. Making this information more understandable and actionable may provide greater health confidence and self-management ability. Ultimately, improving the efficacy of the discharge communication process for patients with ACS and CHF may affect adherence, thereby decreasing morbidity and 30-day readmission rates, an important care quality metric.

\section{Aims and objectives}

We will conduct a pilot, parallel-group, randomised controlled trial to determine the feasibility and acceptability of providing audio-recorded discharge instructions to patients discharged from an inpatient cardiology service. We will also explore the impact of audio recording on exploratory outcomes associated with self-management.

Specific aims:

1. Determine whether providing audio-recorded discharge instructions as a supplemental discharge communication tool will be feasible for cardiology providers and patients to use during the inpatient discharge discussion.

2. Determine whether patients and their families will use audio-recorded discharge instructions after their hospital discharge to self-manage care.

3. Explore the impact of audio recording on patient understanding of their discharge instructions, health confidence, patient activation, general medical adherence, medication adherence, provider communication ability and postdischarge telephone call rates.

\section{METHODS AND ANALYSIS \\ Study setting}

This pilot trial will be conducted at a single site: the general cardiology inpatient unit at Dartmouth Hitchcock Medical Centre (DHMC), a level I trauma and academic medical centre in Lebanon, New Hampshire, USA.

\section{Eligibility criteria}

Providers: We will recruit patients discharged by associate providers (APs, physician assistants and nurse practitioners). We chose to focus on our pilot on APs because they work most consistently on the inpatient unit and are responsible for creating their patients' discharge instructions. Enrolled APs may perform the discharge discussion alone or may choose to participate in the discharge discussion with the patient's bedside nurse, whichever reflects their preferred discharge style. To be eligible for inclusion, APs and nurses must (1) have previous experience participating in the discharge communication process with cardiology inpatients and (2) be willing to participate in the study including the possibility of having their discharge conversation recorded.

Patients: To be eligible for inclusion, patients must be (1) 18 years of age or older, (2) admitted to the inpatient cardiology unit at DHMC, (3) comfortable reading and writing in English and (4) willing to have their discharge conversation recorded. We will exclude patients (1) without capacity to provide consent to the project, as determined by the research assistant (RA); (2) with dementia, schizophrenia and other psychotic disorders, substance-abuse disorders, uncorrectable hearing or visual impairment, as determined by the discharging provider and (3) being discharged to facilities other than home, or patients living in skilled nursing homes or hospice, as determined by the discharging provider, because they engage far less in self-management. The majority of patients are admitted to this inpatient cardiology unit with either ACS or CHF. Given the exploratory nature of our research, we retained broad inclusion criteria to explore whether audio recordings are feasible, acceptable and potentially more beneficial for patients with particular diagnoses.

\section{Intervention}

Usual care: On the day of their hospital discharge, enrolled patients randomised to the usual care arm 
received a verbal discharge discussion with an AP and written discharge instructions.

Intervention: On the day of their hospital discharge, enrolled patients randomised to the audio recording arm (intervention) received usual care, plus a portable electronic device that contains a re-playable audio recording of the discharge discussion. In addition, participants will have the option to record the discharge discussion on a personally owned smartphone or receive access to the recording online using the Health Insurance Portability and Accountability Act (HIPAA)-compliant Open Recording and Logging Systems (ORALS) ${ }^{12}$ Prior to patient enrolment, the research team and the enrolled APs will be trained on how to use the recording device. Patients will also receive printed instructions detailing how to start, stop and retrieve recordings, and will be asked to demonstrate their use of the recording device to confirm understanding of these instructions prior to discharge.

\section{Recruitment}

Providers: APs and nurses will be invited to learn about the study's purpose during department conferences, via recruitment emails and flyers. Interested APs and nurses will meet with a study team member to complete written informed consent. Those who decline will be asked to provide a reason for their decision.

Patients: When an enrolled AP is working on the inpatient cardiology unit, an RA will meet with the provider and multidisciplinary care team to identify eligible patients scheduled for discharge during the morning discharge huddle at 10 am each Monday through Friday. The RA will approach potential patients to determine their interest, confirm eligibility and complete informed consent. An approximate time for the discharge communication will be confirmed with staff and written on the unit 'whiteboard'. The RA will be paged back to the inpatient unit when the discharging AP and nurse are ready to discuss the instructions at the patient's bedside. The RA will proceed with study activities according to allocation assignment. All patients in the intervention arm will receive their recorded discharge instructions on a simple, reusable, portable MP3 playback device that requires no internet or computer access and will be introduced to two additional options for recording. The RA will return to the patient's bedside at the predetermined discharge time to prepare the recording device(s).

\section{Allocation}

A member of the study team not involved in recruitment and data collection, primary investigator (PI) (PJB) will generate a study arm allocation list for each provider using a block randomisation at the provider level (blocks of four that are balanced in the number of allocations to each arm but randomised in terms of order, eg, ABAB and $\mathrm{AABB}$ ). This method was chosen because of the small sample size and to ensure an equal number of patients were assigned to each study arm. The PI (SS)
RAs, providers and patients will be blind to this allocation process, including the size of each block. After baseline data are collected $\left(\mathrm{T}_{0}\right)$, the RA will open a sealed randomisation envelope to reveal the patient's allocation assignment immediately prior to the discharge discussion.

\section{Study procedures}

Immediately following documentation of informed consent, the RA will collect patient demographics and the $\mathrm{T}_{0}$ baseline assessment. The RA will leave the unit and return to the patient's bedside at the predetermined discharge time or when they are paged by the charge nurse or discharging provider. In both arms, the RA will leave the patient's room during the discharge discussion and will return to complete the $\mathrm{T} 1$ assessment after the discharge discussion is complete, documenting time spent in the discussion.

Usual care arm: Patients will experience the usual discharge communication practice, which includes a bedside verbal review of written discharge instructions by the discharging provider(s) (online supplementary material 1). The APs may conduct this review alone or with the patient's nurse.

Intervention arm: When providers and the patient are ready for the discharge discussion, the RA will place a sign outside the patient's room to alert providers and other caregivers that a recording is in process. The RA will set up one MP3 digital recorder and one backup MP3 digital recorder near the patient's bedside and instruct the patient on how to stop should they choose to do so. If the patient chooses to record the discharge conversation using ORALS, an electronic tablet will be used to automatically record and securely transfer the recording to the HIPAA-compliant server. If the patient chooses to record on their personal smartphone device, the patient will be instructed on when to start and stop that recording. Patients will experience the usual discharge communication practice that includes a bedside verbal review of written discharge instructions by the discharging provider(s) (online supplementary material 1 ), but the conversation will be audio recorded. The RA will leave the room immediately after starting the recording(s) and will return after the discharge conversation ends. The RA will demonstrate the use of the MP3 device and provide instructions (playback and battery charging) for this and other types of recordings (ORALS and smartphone) chosen. The RA will document the type(s) of recordings the patient received.

\section{Data collection, management and analysis \\ Data collection}

Providers: We will obtain demographics from providers who agree to participate via survey, including age, sex, race, ethnicity, years in practice and specialty, and will invite enrolled providers to participate in a $15 \mathrm{~min}$ semistructured interview about their experience using audio recordings after the enrolment is complete. We will 
anonymously document reasons providers declined to participate. We will document time providers spent in the discharge discussion. Enrolled APs will report participating patients' primary diagnosis to the RA.

Patients: At the time of enrolment, and before the discharge discussion, all patients will be asked to complete a baseline survey (on paper) consisting of sociodemographics, health literacy, ${ }^{13} 14$ health confidence questions $^{15}$ and the Patient Activation Measure (T0). ${ }^{16}$ Additional outcome data will be collected on the inpatient unit immediately after the discharge discussion (T1) and by telephone 1 week postdischarge (T2) (table 1). On telephone follow-up, patients in the intervention arm will be asked additional interview questions about their experience using their audio recording device(s). DHMC data analytics will use the electronic medical record to report unit-level aggregate data on sociodemographics and disease burden via the Charlson Comorbidity Index so it may be compared with enrolled patients. ${ }^{17}$

\section{Outcomes}

Participant timeline

The participant timeline is outlined in figure 1.

\section{Sample size}

We plan to recruit 50 adult cardiology inpatients for this pilot trial: 25 patients in the usual care arm and 25 patients in the intervention (audio) arm. This number is based on the time constraint to complete all aspects of the study within 1 year and based on the number of daily discharges, including a conservative estimate of enrolling approximately three patients per week over a 16-week period. Prior to study initiation, we plan to recruit a minimum of five APs who will be routinely present on the inpatient cardiology unit to participate in both arms of the study.

\section{Data management}

Study data will be deidentified and securely managed in accordance with Committee for the Protection of Human Subjects (CPHS) by trained research team members using encrypted services licensed by Dartmouth College, including accessing and maintaining audio recordings on a HIPAA-compliant cloud storage system. (Please see data management plan in online supplementary material 2.

\section{Statistical methods}

\section{Quantitative analysis}

Baseline data: We will compare baseline sociodemographics, patient activation and health literacy data between the usual care and intervention arms using $\chi^{2}$ tests for categorical data (Fisher's exact test', where cell sizes of analysis are below 5) and Student t-tests for variables that are closer to continuous in form.

Primary outcome (aim 1): We will consider audio recordings feasible if $>70 \%$ of eligible providers and patients agree to participate in the study and patients receive the intervention according to a fidelity checklist. We will consider audio recordings acceptable for patients if recording use is $70 \%$ or higher. ${ }^{11}$ We will also compare attrition rates and differences in use according to method selected for listening to recording (MP3, smartphone and ORALS system).

Exploratory outcomes and covariates (aim 2):

Within group analyses.

We will compare within-arm differences in health confidence and 13-Item Patient Actitvation Measure (PAM13) scores before discharge and at 1 week postdischarge using the Wilcoxon Sign Test (continuous data) and a McNemars Test (categorical data).

Between group analysis.

We will compare between arm (usual care vs intervention) differences for continuous (PAM-13, Communication Assessment Tool (CAT), Medical Outcomes Study and Adherence to Refills and Medications Scale) ${ }^{16} 18-20$ and categorical (Health Confidence: Confident vs not confident, CAT top score) outcomes and baseline characteristics, using Mann-Whitney U test and Fisher's exact test, respectively. We will control for provider by using a random effects model. We will compare differences in postdischarge telephone call counts, the reasons for calling and the difference in the proportion of patients who use (read) the written discharge instructions. In the intervention arm, we will explore the differences between patients who listen to their audio recording and those who do not. STATA-15 data analytic and statistical software will be used. A p value of $<0.05$ will be considered statistically significant.

\section{Qualitative analysis}

Interviews with patients and providers (APs and nurses) about their experience using audio recordings in the study will be recorded (when permission is granted) and transcribed. Interview data will be analysed using an iterative, inductive thematic approach. ${ }^{21}{ }^{22}$ Two coders will independently review each transcript line-by-line using the qualitative research software ATLAS.ti, identifying possible descriptive codes then discussing codes to build a shared code structure for analysis, which will be used to code all data. ${ }^{21}$ When coding is complete, a three-person team will meet to discuss the data and codes, developing unifying analytic themes to help interpret the data and summarise the findings. ${ }^{21}$

\section{ETHICS AND DISSEMINATION \\ Data monitoring}

After the review of our data collection methods and monitoring plan with Research Operations at the Heart and Vascular Centre, Dartmouth-Hitchcock, we were not required to form a data safety monitoring committee, given the minimal risk to participants. As a substitute, we chose to engage independent research supervisors at the Heart and Vascular Centre and The Dartmouth Institute for Health Policy and Clinical Practice not directly involved in data collection to intermittently evaluate the progress of the project, fidelity to project protocol and 
Table 1 Study outcomes

\begin{tabular}{|c|c|c|c|c|}
\hline \multirow[b]{2}{*}{ Outcome } & \multirow[b]{2}{*}{ Description } & \multicolumn{3}{|c|}{ Assessment } \\
\hline & & TO & T1 & T2 \\
\hline \multicolumn{5}{|l|}{ Primary } \\
\hline Provider enrolment (feasibility) & $\begin{array}{l}\text { The proportion of eligible cardiology providers (APs and nurses) who agreed to } \\
\text { take part in the research. }\end{array}$ & $X$ & & \\
\hline Patient enrolment (feasibility) & $\begin{array}{l}\text { The proportion of eligible cardiology inpatients who agreed to take part in the } \\
\text { research. }\end{array}$ & $x$ & & \\
\hline Intervention fidelity (feasibility) & $\begin{array}{l}\text { The proportion of cardiology inpatients in the intervention arm that received } \\
\text { the audio-recording intervention with full adherence to a predefined protocol } \\
\text { fidelity checklist. }\end{array}$ & & $x$ & \\
\hline $\begin{array}{l}\text { Patient use of intervention } \\
\text { (acceptability) }\end{array}$ & $\begin{array}{l}\text { The proportion of discharged cardiology patients in the intervention arm who } \\
\text { used the audio recording between the day of discharge and a 1-week follow- } \\
\text { up telephone call initiated by the research team. }\end{array}$ & & & $x$ \\
\hline
\end{tabular}

\section{Exploratory}

\begin{tabular}{|c|c|c|c|c|}
\hline Change in patient activation & $\begin{array}{l}\text { The PAM-13 is a 13-item survey that assesses a person's underlying } \\
\text { knowledge, skills and confidence integral to managing his or her own health } \\
\text { and healthcare. }\end{array}$ & $x$ & $x$ & $x$ \\
\hline Change in health confidence & $\begin{array}{l}\text { The proportion of patients who report high confidence measured using an } \\
\text { adapted version of the Health Confidence Measure with three response } \\
\text { options: 'very confident', 'somewhat confident' and 'not confident'. }\end{array}$ & $x$ & $x$ & $\mathrm{X}$ \\
\hline
\end{tabular}

Change in patient ability to
understand health information
Change in patient general medical
adherence
options: 'very confident', 'somewhat confident' and 'not confident'.

The proportion of patients who report a high ability to understand health
information measured using an adapted version of the Health Confidence
Measure with three response options: 'very understandable', 'somewhat
understandable' and 'not understandable'.
Measured using the MOS measure of adherence. The five-item MOS
measures general adherence to medical advice on a 6-point rating scale ranging from 1 (none of the time) to 6 (all of the time).

Patient assessment of discharging
provider communication ability
Measured using the CAT. The 15-item CAT measures patient perceptions of physician performance in the area of interpersonal and communication skills using a 5 -point rating scale ranging from 1 (poor) to 5 (excellent).

Patient adherence to medications Measured using the ARMS-7. ARMS-7 measures medication adherence and refill behaviours on a 4-point rating scale ranging from 1 (none of the time) to 4 (all of the time).

Use of paper discharge instructions The number of patients who use (read) their paper discharge instructions between the time of discharge and the research team's postdischarge followup telephone interview.

Rate of patient-generated postdischarge telephone calls

The number of patient-generated postdischarge phone calls to the outpatient cardiology clinic or hospital emergency telephone line between the time of discharge and the research team's postdischarge follow-up telephone interview.

$\begin{array}{ll}\begin{array}{l}\text { Content of patient-generated } \\ \text { postdischarge telephone calls }\end{array} & \begin{array}{l}\text { DHMC data analytics will provide a list of all phone calls initiated by enrolled } \\ \text { discharged patients to the outpatient cardiology clinic or hospital emergency } \\ \text { telephone line between the day of their discharge and approximately } 1 \text { week } \\ \text { after discharge. PI SS will review telephone data to categorise and quantify } \\ \text { reasons for calling. }\end{array} \\ \text { 30-day readmission rates } & \begin{array}{l}\text { DHMC data analytics will provide a list of enrolled patients who were } \\ \text { readmitted to the hospital between their date of discharge and } 30 \text { days after } \\ \text { discharge. PI SS will review readmission data and will compare rates between } \\ \text { study arms to aggregate data for the cardiology inpatient unit during the } \\ \text { study period. }\end{array}\end{array}$

AP, associate provider; ARMS-7, Seven-Item Adherence to Refills and Medications Scale; CAT, Communication Assessment Tool; DHMC, Dartmouth Hitchcock Medical Centre; MOS, Medical Outcomes Study; PAM-13, 13-Item Patient Actitvation Measure; PI, primary investigator; T0, day of discharge, before randomisation; T1, postrandomisation, day of discharge, immediately following inpatient discharge discussion; T2, postrandomisation, 1 week after inpatient discharge by telephone. 


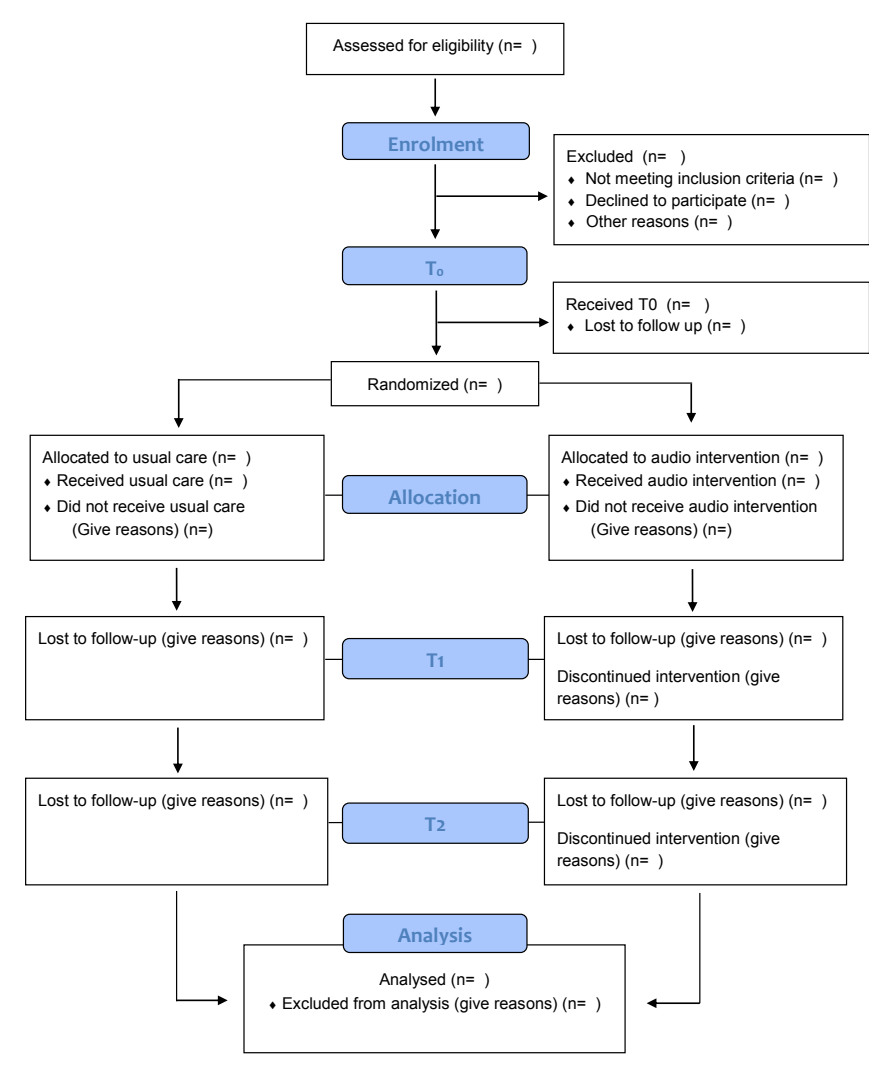

Figure 1 Consort diagram depicting participant timeline and progression through all phases of the study, including final data analysis.

data integrity. Data integrity will be assessed when $25 \%$, $50 \%$ and $75 \%$ of participants are enrolled. After discussion with independent advisors, PIs will decide whether concerns regarding protocol adherence, data collection or provider-patient participation rise to the level of pausing or terminating the trial.

\section{Harms}

Minimal social, legal and psychological risks are anticipated as a result of this study. However, several steps to prevent harm were taken, and channels for reporting and addressing harms were established, including recording only information that reflects standard written discharge instruction, providing education about how to safely maintain and share recorded information and the provision of immediate contact information, including telephone numbers and email addresses for study PIs. Should a data leak occur or unexpected harm be reported, CPHS will be notified immediately and study procedures will cease until an action plan to address a leak is set up in collaboration with CPHS.

\section{Auditing}

The auditing plan includes weekly team meetings to review recruitment, data collection and technical delivery of recordings, a fidelity-to-study procedures checklist captured for every patient, a review of a random selection of $25 \%$ of survey data and audio recordings and observation of the recruitment and intervention process by PIs for five participants.

This research involves human subjects and has received institutional review board approval from the CPHS at Dartmouth College (CPHS\#00031211).

\section{Protocol amendments}

Proposed amendments to the original protocol will be submitted to CPHS at Dartmouth College for approval prior to initiating any changes in study design, recruitment, data acquisition or data analysis. Any protocol amendments will be added to the study record on Clinicaltrials.gov.

\section{Consent or assent}

Written informed consent will be obtained by a member of the research team who has received the following: CITI certification, consent and interviewing training, electronic medical record access courses and audio recording training.

\section{Confidentiality}

Confidentiality of data are maintained by limiting audio recordings to reflect information typically provided in the written discharge instructions. Patients are instructed on how to stop and start recordings during the discharge discussion and on discharge, how to safely maintain and share audio recordings, similar to printed discharge instructions. The research team will store audio recordings, collected survey data and postdischarge telephone call data in Dartmouth-sponsored HIPAA-compliant encrypted systems. All survey, telephone data and qualitative interview data will be deidentified and analysed in aggregate to ensure confidentiality of individual responses.

\section{Access to data}

PIs and research team members at Dartmouth Hitchcock Medical Centre will have access to the final data set. Additionally, deidentified, aggregate data collected using the PAM-SF (PAM-13) will be provided to Insignia Health at the conclusion of the study as part of a licensing agreement to use this measure.

\section{Dissemination policy}

The findings will be disseminated through usual academic channels, including meeting presentations as well as peer-reviewed publications. Written materials and public presentations will not include any personally identifiable data. Based on journal requirements, we will make available a deidentified database of study data.

\section{DISCUSSION}

Findings from this project will contribute to our understanding of the feasibility and acceptability of implementing an inpatient care recording strategy as a tool for communicating critical, often forgotten, discharge information. Our study will also explore the potential of 
sharing recordings with patients to improve self-management, which in turn could contribute to a reduction in high readmission rates reported in cardiology patients. The potential applications of recordings are broad and growing. Advances in novel machine learning and natural language processing techniques open new avenues in medicine, such as automated documentation of clinical notes extracted from the recorded conversations to decrease provider burden. ${ }^{23}$ However, it is important to also consider the privacy and confidentiality implications of recordings, for example, recording and sharing of clinically sensitive conversations and medicolegal concerns of providers. Yet to date, the use of recordings for malpractice suits has not been documented in the small number of clinics where recordings are routinely made available to patients and in at least one case in Arizona, provider participation in recordings may be protective and may result in decreased malpractice premiums. ${ }^{10} 11$ Ownership of the recording is another issue to consider, for example, the application of HIPAA to recordings made and stored by patients (a non-health entity not subject to HIPAA) and not the health system. ${ }^{11}$ A lack of policies to guide the recording and sharing of clinical visits needs to be addressed, ${ }^{10}$ and findings from our project, both quantitative and qualitative, can contribute to this conversation. If managed securely as data in an electronic medical record, recordings could transform healthcare, providing more accurate clinical records and allowing patients and families to revisit medical information that is often forgotten. ${ }^{23}$

Acknowledgements We thank the associate providers and nurses who participated in this study; Dr Mark Creager, Director, Heart and Vascular Center; Dr Jeffrey Kuvin, Section Chief, Cardiovascular Medicine; and Ms Allison Hawke, Research Operations Manager, Heart and Vascular Center, Dartmouth Hitchcock Medical Center.

Contributors All authors have provided substantial contributions to the conception or design of the work, or the acquisition, analysis or interpretation of data.

Funding This work was supported by a Pilot Award from The Hitchcock Foundation \#250-4113.

Competing interests Primary investigator (PI) (SS) and co-investigators (MD, $\mathrm{SD}, \mathrm{JS}, \mathrm{MC}, \mathrm{MB}$ and JOM) have no financial or competing interests to disclose. The PI (PJB) has no financial interests to disclose but does lead a research group, OpenRecordings, that studies sharing audio/video recordings with patients, caregivers and clinicians and has developed the online sharing technology offered in this project (http://www.openrecordings.org/orals).

Patient consent for publication Not required.

Ethics approval The research involves human subjects and has received institutional review board approval from the Committee for the Protection of Human Subjects, Dartmouth College (CPHS\#00031211).

Provenance and peer review Not commissioned; externally peer reviewed.

Data availability statement There are no data in this work.

Open access This is an open access article distributed in accordance with the Creative Commons Attribution Non Commercial (CC BY-NC 4.0) license, which permits others to distribute, remix, adapt, build upon this work non-commercially, and license their derivative works on different terms, provided the original work is properly cited, appropriate credit is given, any changes made indicated, and the use is non-commercial. See: http://creativecommons.org/licenses/by-nc/4.0/.

\section{REFERENCES}

1. Mansukhani RP, Bridgeman MB, Candelario D, et al. Exploring transitional care: evidence-based strategies for improving provider communication and reducing readmissions. P T 2015;40:690-4.

2. Mulhem E, Lick D, Varughese J, et al. Adherence to medications after hospital discharge in the elderly. Int J Family Med 2013;2013:1-6.

3. McDermott K, Elixhauser A, Sun R, Agency for Healthcare Research and Quality. Trends in hospital inpatient stays in the United States 2005-2014, 2017. Available: https://www.hcup-us.ahrq.gov/reports/ statbriefs/sb225-Inpatient-US-Stays-Trends.pdf [Accessed 20 Mar 2019].

4. Kessels RPC. Patients' memory for medical information. J R Soc Med 2003;96:219-22.

5. Chugh A, Williams MV, Grigsby J, et al. Better transitions: improving comprehension of discharge instructions. Front Health Serv Manage 2009;25:11-32.

6. Jack BW, Chetty VK, Anthony D, et al. A reengineered hospital discharge program to decrease rehospitalization: a randomized trial. Ann Intern Med 2009;150:178-87.

7. Dartmouth Hitchcock medical center HCAHPS patient experience: communication scores; 2016.

8. Leventhal R. Discharged with a recording. How Cullman regional is optimizing readmissions work. Healthc Inform 2013;30:41-2.

9. Olives T. Paper ED instructions, Ready, SET, delete. Acad Emerg Med 2012.

10. Barr PJ, Bonasia K, Verma K, et al. Audio-/Videorecording clinic visits for patient's personal use in the United States: cross-sectional survey. J Med Internet Res 2018;20:e11308.

11. Elwyn G, Barr PJ, Castaldo M. Can patients make recordings of medical encounters?: what does the law say? JAMA 2017;318:513-4.

12. Barr PJ, Dannenberg MD, Ganoe $\mathrm{CH}$, et al. Sharing annotated audio recordings of clinic visits with Patients-Development of the open recording automated logging system (ORALS): study protocol. JMIR Res Protoc 2017;6:e121.

13. Chew LD, Griffin JM, Partin MR, et al. Validation of screening questions for limited health literacy in a large Va outpatient population. J Gen Intern Med 2008;23:561-6.

14. Sarkar U, Schillinger D, López A, et al. Validation of self-reported health literacy questions among diverse English and Spanishspeaking populations. J Gen Intern Med 2011;26:265-71.

15. Wasson J, Coleman EA. Health confidence: an essential measure for patient engagement and better practice. Fam Pract Manag 2014;21:8-12.

16. Hibbard JH, Mahoney ER, Stockard J, et al. Development and testing of a short form of the patient activation measure. Health Serv Res 2005;40:1918-30.

17. Huntley AL, Johnson R, Purdy S, et al. Measures of multimorbidity and morbidity burden for use in primary care and community settings: a systematic review and guide. Ann Fam Med 2012;10:134-41.

18. Makoul G, Krupat E, Chang C-H. Measuring patient views of physician communication skills: development and testing of the communication assessment tool. Patient Educ Couns 2007;67:333-42.

19. Hays RD, Kravitz RL, Mazel RM, et al. The impact of patient adherence on health outcomes for patients with chronic disease in the medical outcomes study. J Behav Med 1994;17:347-60.

20. Kripalani S, Goggins K, Nwosu S, et al. Medication nonadherence before hospitalization for acute cardiac events. J Health Commun 2015;20 Suppl 2:34-42.

21. Braun V, Clarke V. Using thematic analysis in Psychology. Qual Res Psychol 2006;3:77-101.

22. Pope C, Ziebland S, Mays N. Qualitative research in health care. analysing qualitative data. BMJ 2000;320:114-6.

23. Elwyn G, Barr PJ, Piper S. Digital clinical encounters. BMJ 2018;361:k2061. 
Correction: Heart sounds: a pilot randomized trial to

determine the feasibility and acceptability of audio

recordings to improve discharge communications for

cardiology inpatients protocol

Schott SL, Dannenberg MD, Dodge SE, et al. Heart sounds: a pilot randomised trial to determine the feasibility and acceptability of audio recordings to improve discharge communication for cardiology inpatients protocol. Open Heart 2019;6:e001062. doi: 10.1136/openhrt-2019-001062

The authors wish to note that Dr. Paul J Barr was the senior author on the project. The correction of this error does not change the results or conclusions of the study, but for clarification, the correct order of authors has been noted below:

Stacey L Schott ${ }^{1}$, Michelle D Dannenberg ${ }^{2}$, Shayne E Dodge ${ }^{1}$, Jesse A Schoonmaker ${ }^{2}$, Molly M Caisse ${ }^{2}$, A. James O'Malley ${ }^{1}$, Martha L. Bruce ${ }^{2}$ and Paul J Barr ${ }^{2}$

${ }^{1}$ Department of Cardiovascular Medicine, Dartmouth-Hitchcock Medical Center, Lebanon, New Hampshire, USA

${ }^{2}$ The Dartmouth Institute for Health Policy and Clinical Practice, Dartmouth College Geisel School of Medicine, Hanover, New Hampshire, USA

Open access This is an open access article distributed in accordance with the Creative Commons Attribution Non Commercial (CC BY-NC 4.0) license, which permits others to distribute, remix, adapt, build upon this work non-commercially, and license their derivative works on different terms, provided the original work is properly cited, appropriate credit is given, any changes made indicated, and the use is non-commercial. See: http://creativecommons.org/licenses/by-nc/4.0/.

C Author(s) (or their employer(s)) 2019. Re-use permitted under CC BY-NC. No commercial re-use. See rights and permissions. Published by BMJ.

Open Heart 2019;6:e001062corr1. doi:10.1136/openhrt-2019-001062corr1

Check for updates 